\title{
Superimposed Quantum Dots: Emerging Optoelectronics
}

\author{
Rostami $\mathbf{A}^{1,2 *}$ \\ ${ }^{1}$ Photonics and Nanocrystal Research Lab (PNRL), \\ Faculty of Electrical and Computer Engineering, \\ University of Tabriz, Tabriz, Iran \\ ${ }^{2}$ SP-EPT Lab, ASEPE Company, Industrial Park of \\ Advanced Technologies, Tabriz, Iran \\ *Correspondling author: Ali Rostami, Photonics and \\ Nanocrystal Research Lab (PNRL), Faculty of Electrical \\ and Computer Engineering, University of Tabriz, Tabriz, \\ 5166614761, Iran; SP-EPT Lab, ASEPE Company, \\ Industrial Park of Advanced Technologies, Tabriz, \\ 5364196795, Iran
}

Received: August 02, 2021; Accepted: August 18, 2021; Published: August 25, 2021

\section{Perspective}

As everybody knows that electron, phonon, and photon transport in solids (crystals) depends on lattice physical properties. Manipulation of propagation properties needs to manipulate crystal parameters such as lattice constant, atoms in the lattice, etc. There are a limited number of crystalline structures in nature to manipulate charge, phonon, and photon transfer in electronics, acoustics, and photonics. The basic problem is how one can make single crystals with desired charge, phonon, and photon transfer performance? Also, how one can manipulate the mechanical, optical, and electrical performance of a device? It seems that nanotechnology and especially nanoparticles and superimposed nanocrystals can help to solve this problem. In this short letter, the superposition of Quantum Dots as a solution to enhance the capability of device designers in this regard is presented, discussed, and demonstrated by simple numerical simulation. If we use the superimposition of QDs, we can realize multi wavelength lasers in a single cavity $[1,2]$. The ultra-broadband semiconductor optical amplifiers can be implemented by this idea [3]. Multi wavelength photodetector with multi-electrical output is another most important application that can be realized using this idea [4]. High-efficiency solar concentrator based on superimposed QDs is introduced in [5]. Other interesting applications can be realized using the proposed idea too. All these advantages are related to optical and electrical properties dependency on the size of nanocrystals [6]. So, it is possible to make different crystals using the superimposition of well-known crystals. To demonstrate that, first, by choosing different crystals, and using the superposition of those, it is shown that the obtained structure is similar to a new crystal with a lattice constant that depends on initial superimposed crystal lattice constants as well as a geometrical combination of those. In the second part, we show that using colloidal QDs, it is so easy to combine different QDs with different sizes in a unique solution and a superimposed QDs with the desired density of each QDs will be available.

Theoretical Analysis- To easily modeling the concept, we assume that a periodic signal shows periodic potential (crystal potential) for real crystals. Then, based on normal methods in solid-state physics, it is so easy to model superimposed QDs. In this case, different gated periodic structures are superposed to make the superimposition of QDs. Finally, using simple mathematical calculation, we show that conclusion of the superimposed QDs is similar to a new periodic structure. On the other hand, using the superimposed QDs with specific distributions, a new single crystal can be achieved. Then electrical, acoustical, and optical properties will be superimposed of basic crystals.

$$
V_{m}(r)=\sum_{n=0}^{\infty} a_{m_{n}} \cos \left(n K_{m} r\right)
$$

where $\mathrm{V}_{\mathrm{m}}, \mathrm{m}, \mathrm{a}_{\mathrm{mn}}, \mathrm{K}_{\mathrm{m}}$ and $\mathrm{r}$ are the potential energy of the crystal, index of QDs, expansion coefficient, lattice vector, and position respectively. In this relation, we assumed that the array of QDs can be approximated by cosine Fourier series expansion and so the first term is enough to approximate that. Using this approximation in the following superposition of $\mathrm{M}$ type array of QDs can be expressed as follows.

$$
V_{\text {total }}(r)=\sum_{j=0}^{M} a_{1_{j}} \cos \left(K_{1_{j}} r\right)
$$

where $V_{\text {total }}, j, a_{1 j}, K_{1 j}, M$, and the $r$ are the superimposed potential energy of the whole crystal, index of lattice, first term of expansion coefficient, lattice vector, number of superimposed QDs, and position respectively. As an example using two types of similar QDs (a little bit different from mole fraction point of view), it is possible to obtain a crystal with a lattice constant that is an average value of two lattices made by QDs. Also, using selective energy contacts, one can access each lattice separately without perturbing others. As it was shown $[1,2]$ using the proposed idea two-wavelength laser using a single cavity is available and this is a new way to integrate optical systems (Figure 1). It should mention that using selective energy contacts, one can populate only one of QDs and so a single wavelength lasing. If two contacts are used, the two groups of QDs can be populated and simultaneously two-wavelength oscillations will have appeared. It can be generalized to have multi wavelength lasing. Also, this idea can be used to fabricate high-efficiency LEDs and white LEDs.

Figure 2 shows schematics of a broadband optical amplifier considering superimposed QDs. To realize superimposed QDs, two main categories of $\mathrm{CdTeS}$ and BiTeS are used. Using solutionprocessed technology different groups of these materials are synthesized and mixed and using spin coating sprayed on the surface. Considering precise optical and electrical analysis, it is shown that the proposed structure has more than $2.5 \mathrm{eV}$ bandwidth [3].

Figure 3 shows the superposition of QDs and a multi wavelength detection system. In this case, three wavelengths are converted to three output electric currents. For separating output electric currents, selective energy contacts are used.

We examined the proposed idea to realize high efficient luminescent solar concentrator [5] and in the following figure, the proposed LSC including superimposed QDs is illustrated. Based on that idea, the efficiency of the LSC was reported more than $30 \%$ (Figure 4). This is amazing and it can be used for home power 

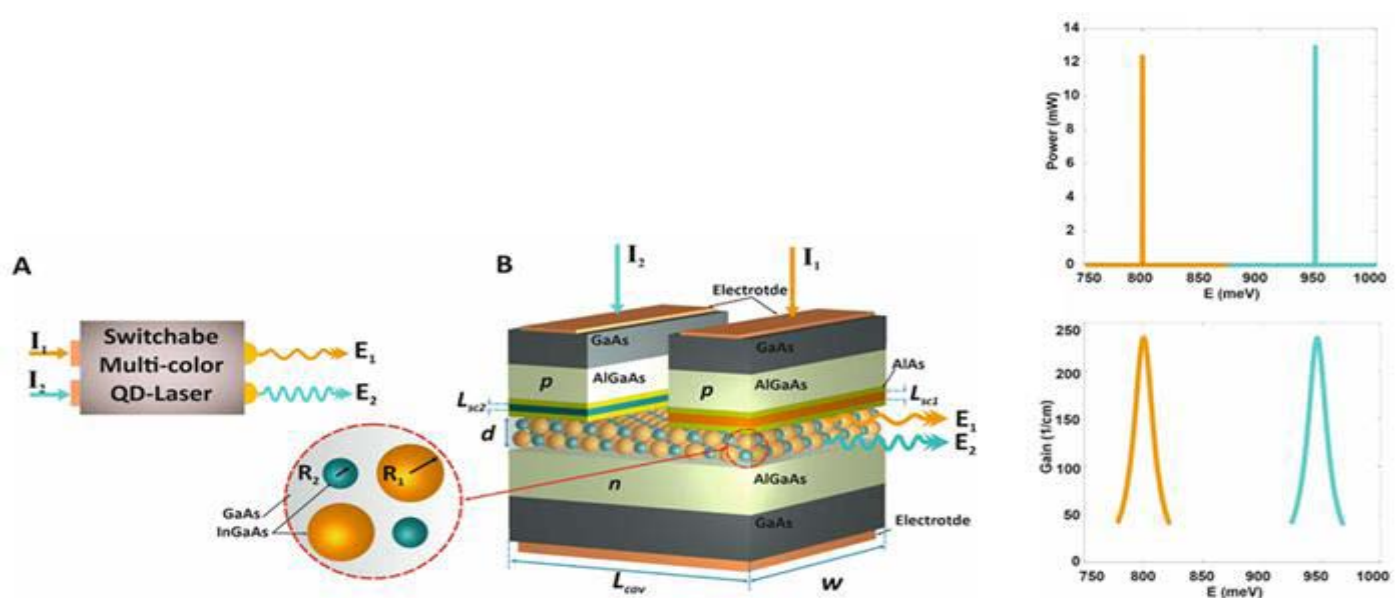

Figure 1: Two-wavelength laser in a single cavity [1]
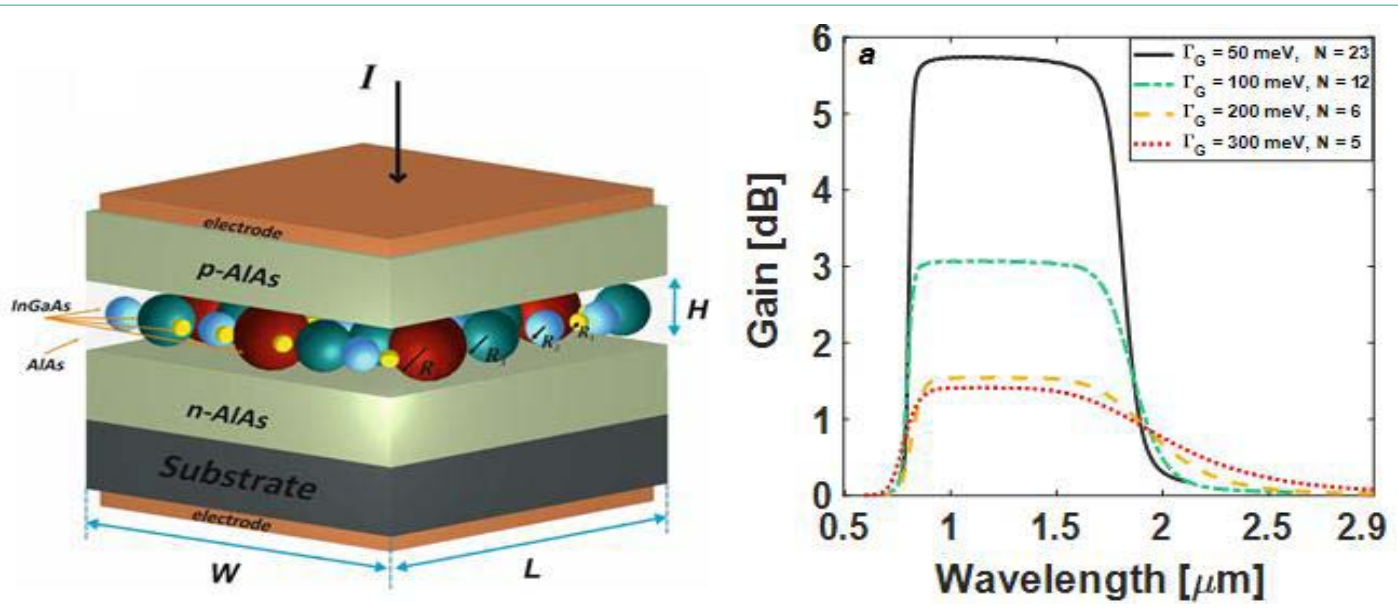

Figure 2: Schematics of superimposed QDs as a broadband optical amplifier [3]

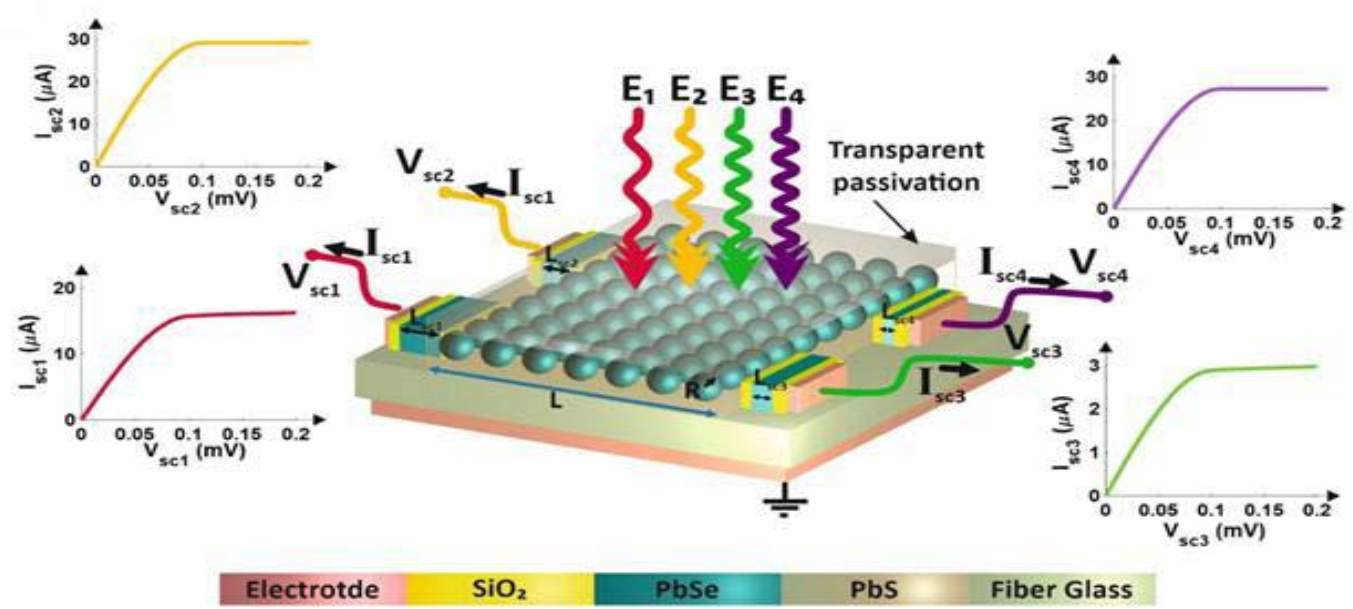

Figure 3: Schematics of superimposed QDs as a multi wavelength photodetector [4] 

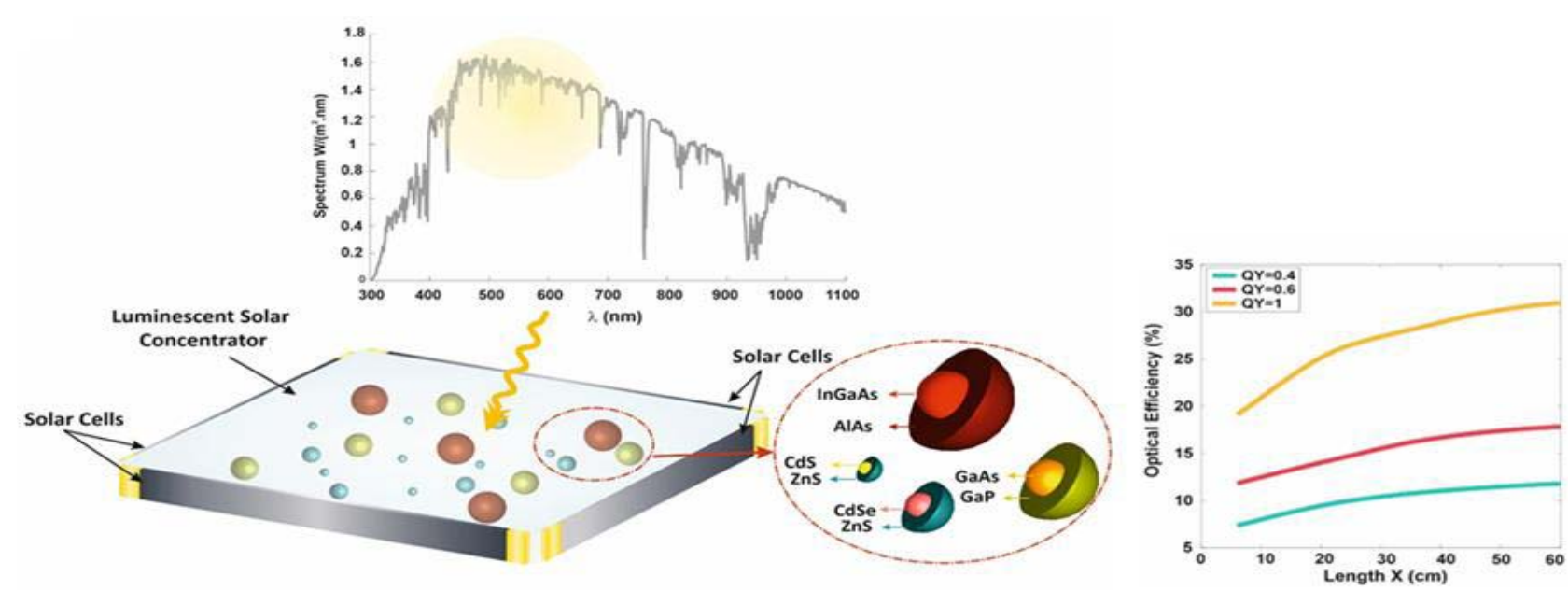

Figure 4: Superimposed QDs based high efficiency luminescent solar concentrator [5].

provided by solar cells in the windows. Since solar power is clean and more interested by energy providers especially for next years, thus the proposed idea can be commercialized by appearing new startups and professional companies.

It should mention that experimentally the proposed idea is feasible and nowadays, solution process technology is mature and able to synthesis QDs with acceptable resolution in size and different materials for core or shell. Using a synthesis of QDs as colloids and then mixing with a specific concentration a superimposed colloid can be obtained and it can be spin-coated on a surface or can be used to make composite materials. Thus, the proposed idea is reliable and a lot of applications can be produced based on the idea. In conclusion, it should mention that the superimposed quantum dots is a new idea to develop a lot of interesting applications, especially in optoelectronics. A few applications in this paper were mentioned and there is valuable potential in this area to create interesting applications.

\section{References}

1. S Matloub, P Amini, A Rostami. "Switchable multi-color solution-processed QD-laser". Scientific reports. 2020; 10: 1-14.

2. P Amini, S Matloub, A Rostami. "Multi-wavelength solution-processed quantum dot laser". Optics Communications. 2020; 457: 124629.

3. HG Yousefabad, S Matloub, A Rostami. "Ultra-broadband optical gain engineering in solution-processed QD-SOA based on superimposed quantum structure". Scientific reports. 2019; 9: 1-11.

4. M Tahmasebi, A Rostami, S Matloub, H Mirtagioglu. "Selective Energy Contacts and Multi-Wavelength Solution-processed Quantum Dot Infrared Photodetector". IEEE Journal of Quantum Electronics. 2021

5. MR Mirzaei, A Rostami, S Matloub, H Mirtaghizadeh. "Ultra-high-efficiency luminescent solar concentrator using superimposed colloidal quantum dots". Optical and Quantum Electronics. 2020; 52: 1-16.

6. Jianwei Cai and GD Mahan. "Transport properties of quantum dot arrays". Physical review. B, Condensed matter. 2008; 78: 35115. 